\title{
Effects of charmed particles in Extensive Air Showers
}

M. A. Müller*

Instituto de Física e Matemática, Universidade Federal de Pelotas/UFPEL, Pelotas, RS, Brazil and Instituto de Física, Universidade Federal do Rio de Janeiro/UFRJ, Rio de Janeiro, Brazil

E-mail: mamullereif.ufrj.br

\section{Victor P. Gonçalves}

Instituto de Física e Matemática, Universidade Federal de Pelotas/UFPEL, Pelotas, RS, Brazil

E-mail: barros@ufpel.edu.br

Charmed particles are rare in Extensive Air Showers (EASs), but their effects can be radical in the EASs development. If such particles carry a large fraction of primary energy, they can to reach large atmospheric depths, depositing their energy in deeper layers of the atmosphere, changing the EAS observables $\left(X_{\max }, R M S\right.$ and $N_{\max }$ ) and the shape of the longitudinal profile of the energy deposited in the atmosphere. Very energetic charmed hadrons may be produced in the upper atmosphere when a primary cosmic ray or a leading hadron in the EASs collides with the air. Since the inelasticity in these collisions is much smaller than in proton and pion collisions, we will have rare events in which a heavy hadron particle transport a significant amount of energy deep into the atmosphere. A heavy particle's energy deposition relatively near the ground produces muons and other particles that could change significantly the EASs longitudinal profile seen in the Fluorescence Detector and/or the temporal distribution and signal observed in the ground detectors. In this contribution we consider a modified version of the EAS simulator, CORSIKA, which takes into account the production of charmed particles in the first interaction of the primary cosmic ray. The heavy charmed particles are produced via Intrinsic Quark Model (IQM) model. We present results for the EAS longitudinal profile and lateral distribution of particles, and analyze the implications in the EAS reconstruction parameters.

36th International Cosmic Ray Conference -ICRC2019-

July 24th-August 1st, 2019

Madison, WI, U.S.A.

* Speaker. 


\section{Introduction}

The precise description of the Extensive Air Showers (EASs) is fundamental for the Cosmic Rays Physics. Primary particles reach the Earth with energies up to $10^{20} \mathrm{eV}$, which are well above the ones reached at colliders. Therefore, the simulation of these EASs requires the extrapolation of the known physics. Very energetic charm an bottom hadrons may be produced in the upper atmosphere when a primary cosmic ray or a leading hadron in the EASs collides with the air. Because of their short mean life, $\approx 10^{-12} s(\approx 300 \mu \mathrm{m})$, heavy hadrons decay before interacting. At $E \gtrsim 10^{7} \mathrm{GeV}$ heavy hadrons reach their critical energy and their decay probabilities decrease rapidly. At $E \approx 10^{8} \mathrm{GeV}$ their decay lenght becomes of order $\approx 10 \mathrm{~km}$, implying that they tend to interact in the air instead of decaying. Since the inelasticity in these collisions is much smaller than the one in proton and pion collisions, the heavy hadrons keep a higher fraction of their energy after each interaction. Consequently, rare events can be present in the EASs, where a heavy hadron particle transport a significant amount of energy deep into the atmosphere, giving rise to additional contributions to the development of EAS. Heavy particles can be produced at any stage of the EAS development, but it is mainly during the first interaction of primary that they are produced with a significant fraction of primary energy. Another important aspect is that the collisions of heavy hadrons with air are very elastic. For example, a $D$ meson after a $10^{9} \mathrm{GeV}$ collision can keep around $55 \%$ of the initial energy, whereas a $B$ meson will have typically $80 \%$ of the incident energy after colliding with an air nucleus [1]. If heavy hadrons are produced with a high fraction of the primary particle, they will interact rather than decaying. If several elastic interactions occur, these heavy hadron particles can transport a significat amount of energy deep into the atmosphere and likely have observable effects on the EAS development. These EAS effects will be discussed in this work.

In this contribution we investigate the impact of charmed particles in the EASs development using a modified version of the CORSIKA [2] (Cosmic Ray Simulations for Kaskade) Monte Carlo, which includes the charm and bottom particle production in the first interaction of the primary particle $[3,4]$ (denoted hereafter HQ CORSIKA). For comparison, we have present the predictions of the standard version of CORSIKA (denoted STD CORSIKA), which disregards the presence of the charm and bottom particles. In the next section, we explain the main details of the HQ CORSIKA. In both codes (standard CORSIKA and HQ CORSIKA) we will assume the QGSJET01 model to describe the high energy hadronic interactions. In Sections 3, 4 and 5 we present our results for the longitudinal profile, depth and lateral distribution, respectively. In Section 6 we discuss our main results and, finally, in Section 7 we present our conclusions.

\section{Heavy particle simulation}

In order to estimate the impact of charmed hadrons in the EASs development we have modified the CORSIKA Monte Carlo by including the production of these particles in the proton - air collision at the first interaction of primary cosmic ray or in the shower if the proton emerge from the first interaction with an incident energy $>3 \times 10^{17} \mathrm{eV}$. These particles are produced considering the Intrinsic Quark Model (IQM), which takes into account the probability that the an intrinsic charm can be present in the projectile wave function. Such model allows to estimate the probability for the quark charm to be contained in different species of hadrons after $p-$ Air collision [5]. 

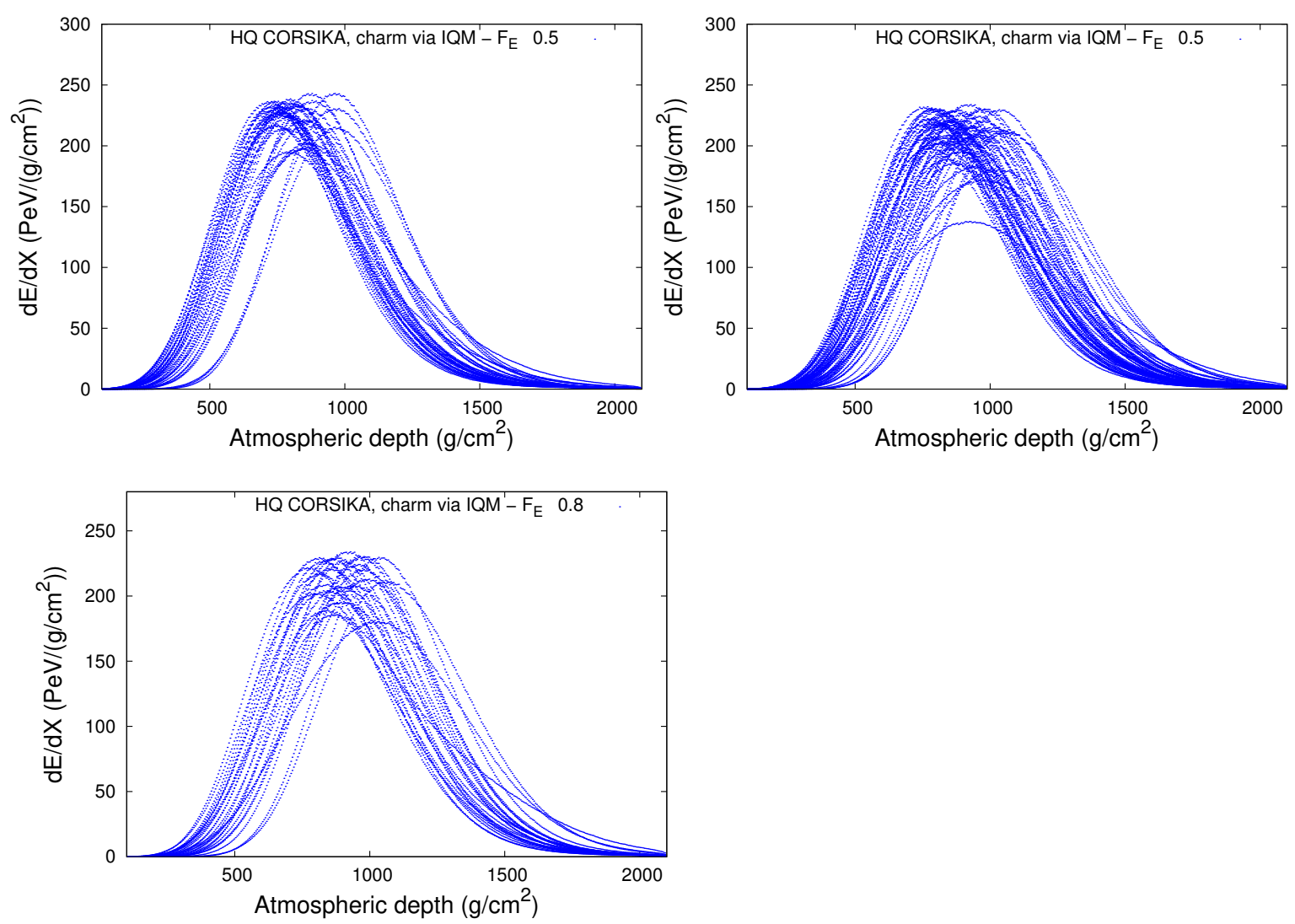

Figure 1: Longitudinal profiles for the energy deposited in the atmosphere. Results for $F_{E}<0.5$ (upper left), $F_{E} \geq 0.5$ (upper right) and $F_{E} \geq 0.8$ (lower panel).

The standard version of the CORSIKA does not explicitly include the interaction of charm and bottom particles. In our analysis we include this possibility and take into account of the production and propagation of heavy particles using the DPMJET and QGSJET models to describe the interactions. For this analysis, we only consider production of the following states $\Lambda_{c}^{+}, D^{0}, D^{+}, D_{s}^{+}, B^{+}$ and $B^{0}$ (and their anti-particles) [3, 4].

\section{Longitudinal profiles}

In this section we will analyze the longitudinal profiles of total energy deposited in the atmosphere considering $\approx 150$ EAS in our study. We will assume that the primary is a proton with an energy of $1 \times 10^{20} \mathrm{eV}$ with a zenithal angle of $60^{\circ}$. The reason behind choosing this zenithal angle is that any effect due to the production of heavy hadrons will only be noticeable deep in the atmosphere. Since the early stages of the EAS longitudinal development are almost insensitive to the heavy hadron propagation, we should to consider EASs with larger zenithal angles in order to observe the late longitudinal development. In our study the production of charm heavy particles will be modelled using the IQM model. For comparison we will present the HQ CORSIKA and STD CORSIKA predictions. 

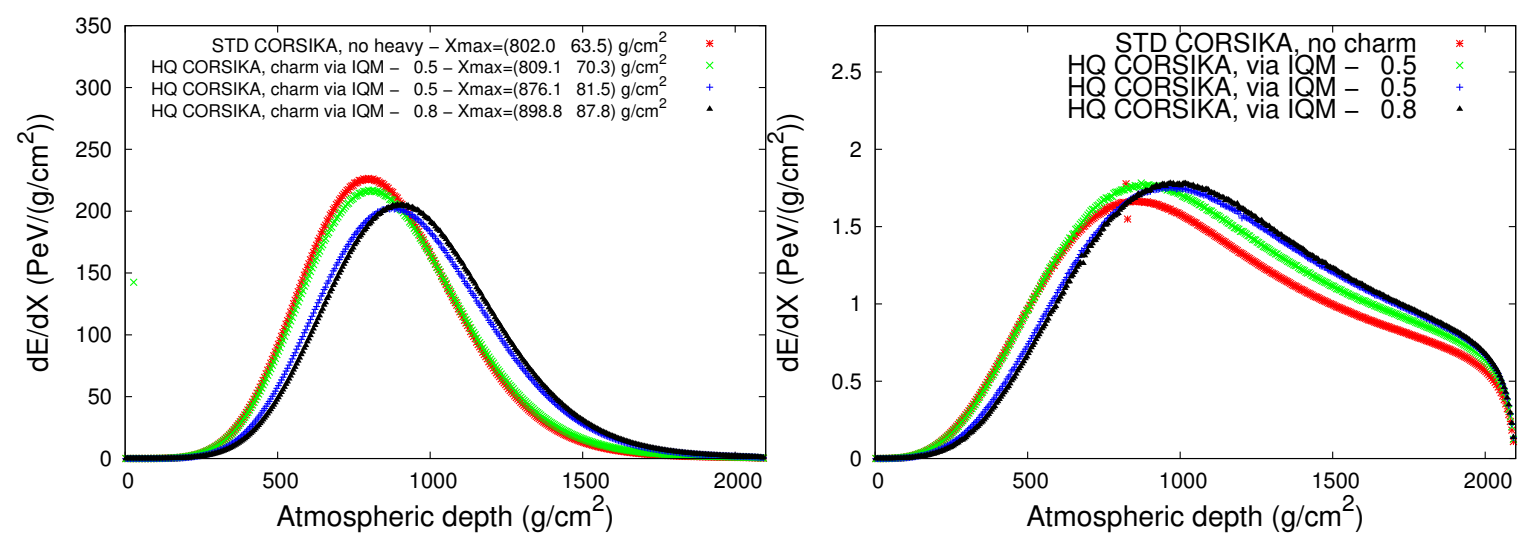

Figure 2: Average longitudinal profiles of energy deposited in the atmosphere. Results for the total (left panel) and muon (right panel) energy deposited are presented considering the STD CORSIKA and HQ CORSIKA models. Predictions for $F_{E}<0.5, F_{E} \geq 0.5$ and $F_{E} \geq 0.8$ are presented separately.

In order to estimate the impact of the charmed particles in the EASs, let's separate our results according to the energy fraction $\left(F_{E}\right)$ carried by heavy particle produced at the first interaction. We will present results for $F_{E}<0.5, F_{E} \geq 0.5$ and $F_{E} \geq 0.8$. Such separations allows to analyse the longitudinal profile for rare charmed events. In Fig. 1 we present our predictions. We have that for $F_{E} \geq 0.5$ and $F_{E} \geq 0.8$ the EASs profiles are significantly modified, including changes in the shapes of the profile and the $X_{\max }$ depth. In particular, for $F_{E} \geq 0.8$ we have more radical effects at the EASs development. Heavy secondaries producion via IQM charm production have higher energy fractions of primary energy, thus carrying a larger amount of energy deep into the atmosphere. For $F_{E} \geq 0.8$, the secondaries have an energy of $2.4 \times 10^{10} \mathrm{GeV}$, with their decay lenght beings of order of $\approx 1000 \mathrm{~km}$. It implies that they tend to interact in the air instead of decaying, implying additional contributions to the EASs development.

Using these previous results, we can estimate the average longitudinal profile. The results are presented in Fig. 2. We can see that the presence of charmed particles implies a significant modification in the average longitudinal profiles. In particular, if the energy fraction of heavy particles is increased, we have a deeper $X_{\max }$ and a higher $R M S$. We can also observe a significant change in the profile, depending of the energy fraction. At higher fractions of energy produced $\left(F_{E} \geq 0.8\right)$, the average longitudinal profile is more modified. For $X_{\max }$ we have a shift to deeper layers of the atmosphere in relation to the STD CORSIKA, with the relative difference being about $12 \%$. On the other hand, for the $R M S$, we have a relative difference of about $40 \%$ [4]. For the charm production with $F_{E} \geq 0.8$ we find the values of $898.8 \mathrm{~g} / \mathrm{cm}^{2}$ and $87.8 \mathrm{~g} / \mathrm{cm}^{2}$, respectively, for $X_{\max }$ and $R M S$. We can also observe a significant change of the shape of the profile, with the deposition of less energy according to the energy fraction. The interaction of charmed particles are more elastic than other particles, which implies that the charmed particles produced with a higher primary fraction will deposit energy more slowly in the atmosphere and can carry larger energies deeper in the atmosphere. For higher fractions of energy $\left(F_{E} \geq 0.8\right)$, the changes in the average longitudinal profiles has its greatest effect. Regarding the $R M S$, we have larger values, which means that the depth of the maximum of EAS $\left(X_{\max }\right)$ fluctuation is bigger, which also is 

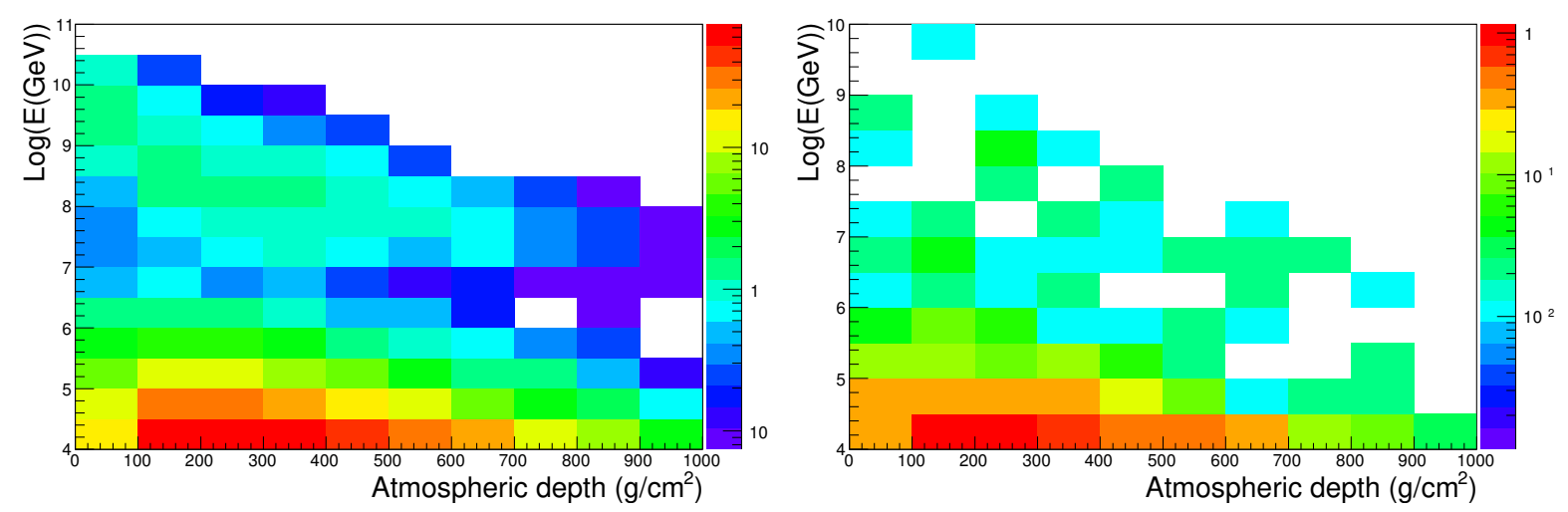

Figure 3: Evolution in the atmosphere of the secondary particles produced in the EAS. The particles are separated according the level that they are produced in the EAS development, from the first interaction down to the sea level. Results for the average number of secondary charmed particles (muons) are presented in the left (right) panel.

associated to the fact that interaction of heavy particles is more elastic. All these EAS effects $\left(X_{\max }\right.$ shift, larger $R M S$ and more elongated shape of the EAS profiles) can change significantly the EASs longitudinal profile seen in fluorescence telescopes and/or the temporal distribution observed in the surface detectors. In fact, we could see some double core profiles. The global effect of all these changes in longitudinal profile is the possible lower energy reconstructed of the EAS and higher uncertainties. The $X_{\max }$ and $R M S$ are directly linked to the mass composition of the primary cosmic ray. The appearance of charms in the EAS makes it more difficult to make such a connection, because of the deeper $X_{\max }$ and larger $R M S$.

\section{Charm Production Depth}

In this section we investigate the production depth of the charmed particles generated during the EASs develpment as well as of the secondary muons, which are produced from the charm decay. We will analyze $\approx 150$ vertical EASs characterized by a primary proton with energy of $3 \times 10^{19} \mathrm{eV}$. We will use the HQ CORSIKA with charm production via IQM model [6]. In Fig. 3 (left panel) the charm particles are separated according the position where they are produced during the EAS development, from the first interaction of the primary down to the sea level, in bins of $100 \mathrm{~g} / \mathrm{cm}^{2}$ of atmospheric depths and half of a energy decade. One have that the total number of charmed particles produced in all bins, with energy higher than $10^{4} \mathrm{GeV}$, are $\approx 600$ charms per EAS, being $\approx 200 D^{0}, \approx 180 D^{+}, \approx 3 D_{s}$ and $\approx 200 \Lambda_{c}$. Moreover, $\approx 20$ charms are produced with energy higher than $10^{8} \mathrm{GeV}$. We can see that most part of charm particles produced have energy below $10^{6} \mathrm{GeV}$ and are being produced between 100 and $500 \mathrm{~g} / \mathrm{cm}^{2}$. On the other hand, the charmed particles with higher energy are produced in the beginning of the atmosphere, with a depth less than $200 \mathrm{~g} / \mathrm{cm}^{2}$. The average number of charmed particles produced above $10^{6} \mathrm{GeV}$ is $\approx 37$, which will decay and produce high energy muons and neutrinos that will reach the ground. The corresponding results for muons are presented in Fig. 3 (right panel). 

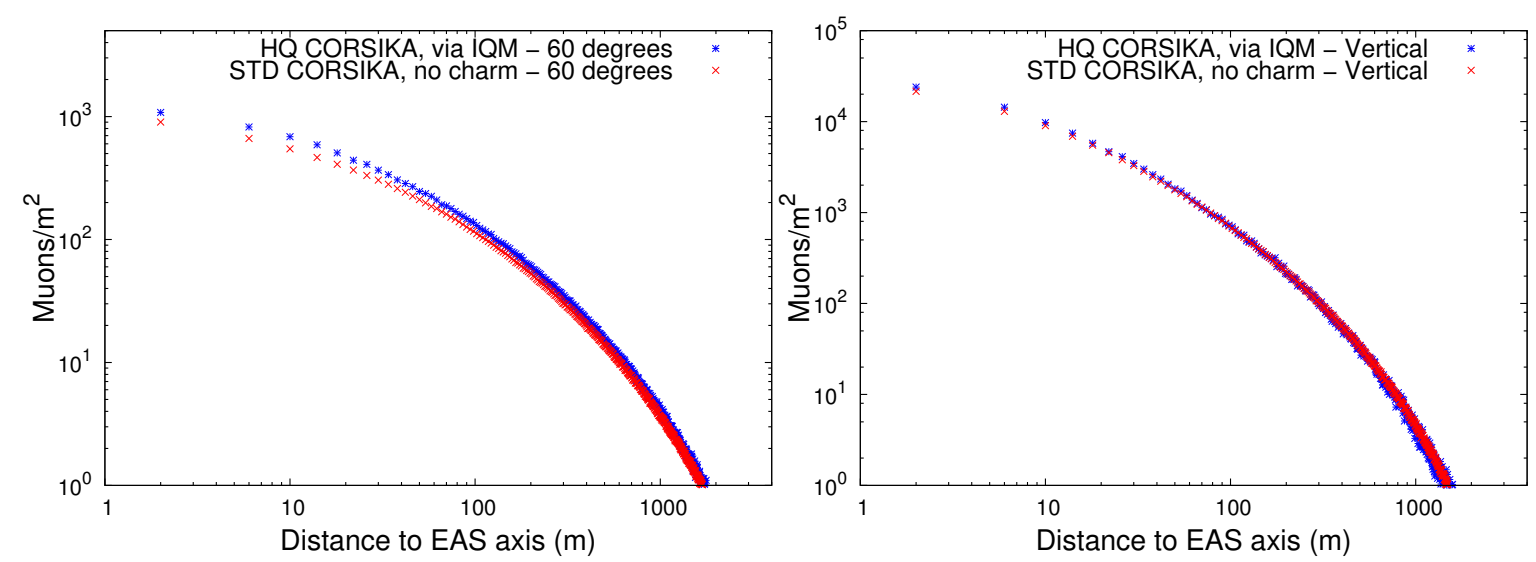

Figure 4: Lateral distribution of the muon density considering vertical (right panel) and EASs with a zenithal angle of $60^{\circ}$ (left panel).

\section{Lateral Distribution of Muons}

In this section we will investigate how the presence of charmed particles in the development of the EASs modify the lateral distribution of muons at ground. We will consider $\approx 100$ EASs characterized by a proton as the primary cosmic ray, which incides in the atmosphere with energy of $3 \times 10^{19} \mathrm{eV}$. We will consider vertical showers as well as EASs with a zenithal angle of $\theta=$ $60^{\circ}$. The Fig. 4 presents our results for the lateral distribution of muons density according to distance from EAS axis. For EASs with $\theta=60^{\circ}$, we have an excess of $\approx 20-30 \%$ in the muon density. On the other hand, for a vertical shower, the excess is of $\approx 15 \%$ to distances close to the EAS axis (below $300 \mathrm{~m}$ ), ie, for muons with large longitudinal momentum and small transverse momentum. The Intrinsic Quark Model implies that the produced charmed particles have high energies, reaching $3 \times 10^{19} \mathrm{eV}\left(F_{E} \approx 1\right)$ in some cases. This larger values of secondary charmed particles cause a modification in the lateral distribution of the muons. We observe that the lateral distribution is different for vertical and $\theta=60^{\circ}$ EASs. In particular, the number of muons is larger to $\theta=60^{\circ}$ EASs. More inclined EASs cross more atmosphere. For $\theta=60^{\circ}$, they cross $2100 \mathrm{~g} / \mathrm{cm}^{2}$. Thus, more inclined EASs have more muons coming from the decay of charmed particles that reach the ground more spread. The impact of the charmed particles in the muon density can be estimated by calculating the ratio between the HQ CORSIKA and STD CORSIKA predictions, which will be denoted by $\rho_{\mu}$ hereafter. The results are presented in Fig. 5. We can see that $\rho_{\mu}$ decreases with the distance of the EAS axis, with the muons being mainly produced close to the EAS axis. It means that the muons coming from charm particles are concentrated in that EAS region.

\section{Discussion}

The propagation of energetic heavy hadrons in the EASs modifies the characteristics of the events as detected by the ground detectors. Since charmed particles are able to reach larger depths in the atmosphere, interacting rather than decaying, they delay the EAS development. The resulting longitudinal profiles are wider, have more particles at ground level and larger depths [3, 4]. In 

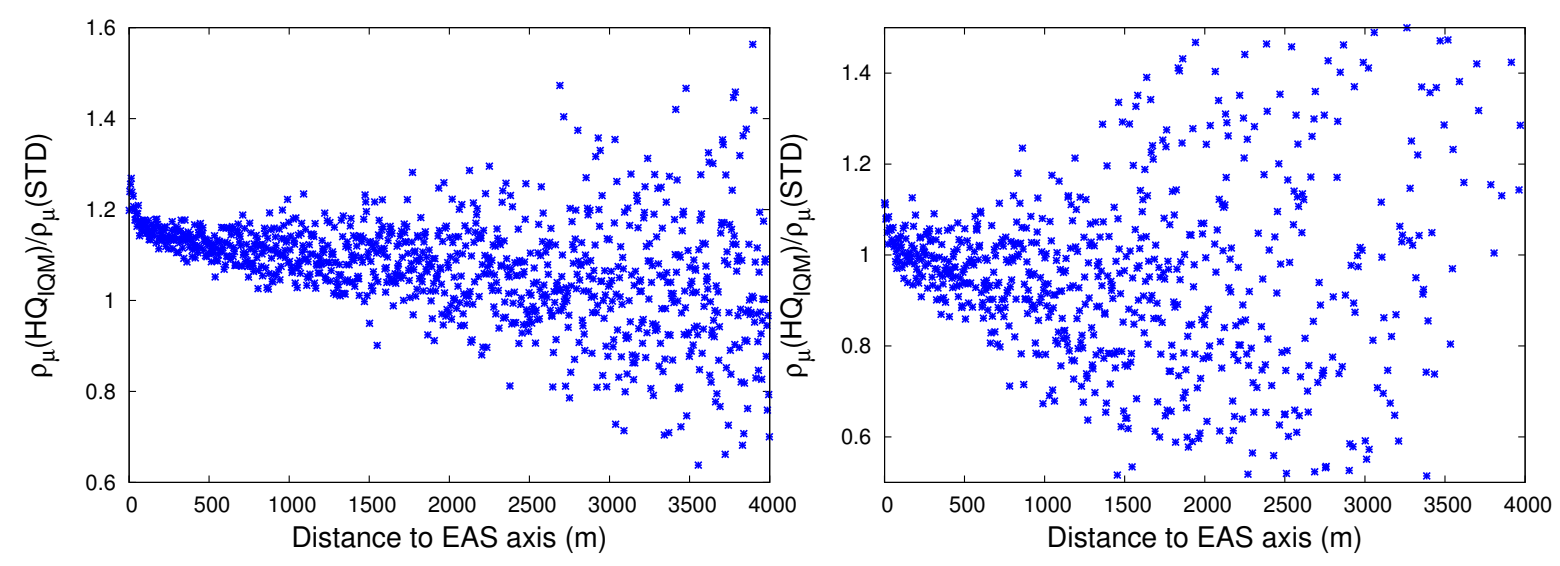

Figure 5: Ratio between the $H Q$ and STD CORSIKA predictions for the lateral density distribution of muons considering vertical (right panel) and EASs with a zenithal angle of $60^{\circ}$ (left panel).

addition, EASs that transport energy deeper in the atmosphere result in a larger number of electromagnetic component inside the Water-Cherenkov tanks. In inclined EASs, this means that detectors that would otherwise only detect muons, since the electromagnetic component is largely absorbed, will still have a significant electromagnetic component. This fact can cause for instance, broader signals, with larger average fall-times and rise-times [7].

Regarding to the average profiles, the presence of charmed particles implies a significant modification of the longitudinal profiles. With the increasing of the energy fraction carried by the heavy particles, we have a deeper $X_{\max }$ and a higher $R M S$. We can also observe a significant change in the profile curve, with the deposited energy being smaller for larger energy fractions. The change in the average longitudinal profiles is larger for the higher fractions $\left(F_{E} \geq 0.8\right)$. For $X_{\max }(R M S)$ we have a shift to deeper layers of the atmosphere in relation to STD CORSIKA, with the relative difference being about $12 \%(40 \%)$.

Concerning the lateral distribution of muons, our results indicate that the presence of charmed particles implies an excess in the muon density of order of 20-30 \% for EAS with $\theta=60^{\circ}$. For vertical EASs the excess is $\approx 15 \%$ to distances close to the EAS axis (below $300 \mathrm{~m}$ ), ie, for muons with large longitudinal momentum and small transverse momentum. The charmed particles generated by the IQM carry a higher energy fraction, which implies the modification of the lateral distribution of muons in the EAS. We observe that the impact is different for vertical and inclined EASs, with the number of muons being larger for $\theta=60^{\circ}$ EASs. Thus, more inclined EASs has more muons coming from the charm decay, which reach the ground more spread.

\section{Conclusions}

The production and propagation of heavy hadrons are expected to modify the EAS development. In this contribution we have presented our results for several EASs observables obtained using a modified version of the CORSIKA Monte Carlo, which includes the production and propagation of charmed particles. We have considered the Intrinsic Quark Model for the description of the charm production, which takes into account the probability of an intrinsic heavy flavour in 
the hadronic wave function. The presence of charmed particles implies that the $X_{\max }$ is shifted to deeper atmospheric layers and that a larger number of particles is present deep in the atmosphere (mainly muons). Since the early stages of the EAS longitudinal development are not sensitive to the heavy hadron propagation, we must to consider EASs with larger zenith angles if we want to observe the late longitudinal development. Moreover, our results indicate that if charmed particles are produced in the EAS development, its front hitting the ground detectors will be modified. In particular, the change will be larger if the charmed particles are produced with a large energy fraction of the primary and the shower is very inclined. In this case, we have a larger impact in the EAS parameters, which could be a possible source of the anomalous events observed for example in the Pierre Auger Observatory.

\section{Acknowledgments}

We would like to thank Alberto Gascón for the productive discussion. This work was partially financed by the Brazilian funding agencies CNPq, CAPES, FAPERGS and INCT-FNA (process number 464898/2014-5).

\section{References}

[1] C. A. García, et al, Production and propagation of heavy hadrons in air-shower simulators, Astroparticle Physics, 46, 29(2013).

[2] Heck, D., Knapp, J., Capdevielle, J. N., Schatz, G., and Thouw, T., Report FZKA 6019 (1998), Forschungszentrum Karlsruhe, Germany.

[3] A. Bueno and A. Gascón, Corsika implementation of heavy quark production and propagation in Extensive Air Showers, Computer Physics Communications, 185, 638 (2014).

[4] M. A. Müller and V. P. Gonçalves, Longitudinal profiles of Extensive Air Showers with inclusion of charm and bottom particles, International Journal of Modern Physics A, 34, 1950069 (2019).

[5] A. Gascón and A. Bueno, Charm production and identification in EAS, Gap Note (Internal notes of Pierre Auger Collaboration), 2011-019 (2011).

[6] M. Muller and V. P. Gonçalves, Prompt muons and neutrinos in simulations of Extensive Air Showers, in preparation.

[7] A. Gascón HEAVY QUARK SIMULATION AND IDENTIFICATION AT THE PIERRE AUGER OBSERVATORY, $\mathrm{PhD}$ Thesis, 2013. 